\title{
Synthesis of Graphene Oxide from Waste Carbon Tyre using Modified Hummer's Method
}

\author{
N.N. Bonnia ${ }^{1 *}$, A.Z. Zanuri ${ }^{1}$, N.A. Asli ${ }^{1,2}$, N.A. Masdar $^{2}$, S. Ratim ${ }^{1}$, S.M. Yahaya ${ }^{1}$, M.M. Mahat ${ }^{1}$, R. Ramli ${ }^{1}$ \\ ${ }^{I}$ School of Physics and Material Studies, Faculty of Applied Sciences, Universiti Teknologi MARA, 40450 Shah Alam, \\ Selangor, Malaysia \\ ${ }^{2}$ NANO-SciTech Centre, Institute of Science, Universiti Teknologi MARA, 40450 Shah Alam, Selangor, Malaysia \\ *Corresponding author E-mail: noornajmi@ salam.uitm.edu.my
}

\begin{abstract}
Recently, graphene was produced from graphite powder using chemical vapour deposition (CVD) or Hummer's method. Graphene is widely used in many applications and give a lot of advantages for industry. In this study, graphene oxide was synthesized from waste carbon tyre using modified Hummer's method. This green technology turned waste material to wealth. The morphology and structural properties of the graphene oxide were investigated using Raman spectroscopy, scanning electron microscopy (SEM) and energy dispersive X-ray spectroscopy (EDX). Raman analysis was confirmed that graphene oxide was successfully synthesized from waste carbon tyre. It was confirmed the peaks shows that $\mathrm{D}$ band and $\mathrm{G}$ band was at $1361 \mathrm{~cm}^{-1}$ and $1596 \mathrm{~cm}^{-1}$ with the intensity ratio of the $\mathrm{D}$ band relative to the $\mathrm{G}$ band $\left(\mathrm{I}_{\mathrm{D}} / \mathrm{I}_{\mathrm{G}}\right)$ is 0.88 . The formation of few sheets of grapheme oxide that stalked together on the surface of the sample structure, bumping pieces and coarse surface was confirmed by scanning electron microscopy (SEM). The elemental composition of carbon (C) is $50.90 \%$ and oxygen $(\mathrm{O})$ is $49.10 \%$ which showed a good composition for graphene oxide. All the results were confirmed that the graphene oxide has been synthesized from waste carbon tyre using modified Hummer's method which next will forms graphene powder through exfoliation method.
\end{abstract}

Keywords: Graphene; Graphene oxide; Modified Hummer's method; Nanomaterials; Waste carbon tyre

\section{Introduction}

Graphene is light and strong material compared to other material and these unique properties can be used for various applications. Graphene has been widely known in aerospace industries due to it opposes properties such as strong, stiff but very light in weight. The aerospace engineers are composed material by carbon fiber for the production of aircraft as it is very strong and light weight. These characteristics can also improve the high strength requirement applications such as body protector for military personnel and vehicles. Graphene nanomaterial is widely used nowadays in many field of research. Nanomaterials have unique chemical and physical properties and have made them valuable additions to many domestic and industrial applications [1].

Graphene is a material that having $\mathrm{sp}^{2}$ hybridization carbon atoms and has fascinated much attention in recent years owing to its extraordinary properties [2]. Graphene can be extracted from graphite powder by many types of method and at the same time, graphene shows good properties such as having high mechanical strength, light weight, best electrical and thermal conductivity $[3,4,5]$. Graphene has been synthesized by both top down and bottom up approaches [6]. Many researchers have attracted much on graphene due to its high aspect ratio and desirable mechanical, thermal and electrical properties [7]. The typical precursor for synthesis graphene before using natural carbon sources is methane, acetylene or alcohol. This precursor consists toxicity and expensive. Several attempts have been devoted to synthesis graphene from carbon sources that non-toxic and non-explosive compare to chemical. There is some possibility to obtain the graphene from carbon source without catalyst and chemical treatment.

Environmental issues are becoming increasingly prominent area of research due to usage of hazardous substances. The challenges being faced today in commercializing graphene are how to produce high quality material, on a large scale at low cost and in a reproducible manner. Furthermore, the technique that currently used to synthesis graphene is often complex, tedious and release high level of toxicity. Therefore, there is need to develop graphene oxide to produce graphene by following environmentally friendly approaches with low cost of production.

Chemical reduction is one method to obtain great amount of graphene from graphite oxide. The oxidation of graphite is performed to synthesis graphene oxide and can be done by using few types of oxidants for instance, concentrated sulphuric acid, potassium permanganate or nitric acid [3]. The major issue with CVD method is the precursor. The usage of toxic and explosive requires a growth system to avoid any harmful and be safe. Mechanical exfoliation is the oddest method to extract monolayer graphene flakes on substrate and this formation of graphite is done where there is a stacking of single atomic layers by poor van der Waals forces and carried out by electric field, ultra-sonication, transfer printing method and scotch tape [3]. However, graphene synthesized from mechanical exfoliation having poor bonding and big lattice spacing in vertical direction.

Graphene oxide was firstly synthesized by Brodie method in 1859 . One more methods to produce graphene is from Hummer's method. Hummer's method is cost effective and scalable preparation [8]. Modified Hummer's method was used to obtain graphene oxide-monohydrated manganese phosphate composite [9]. Modi- 
fied Hummer's method also used to synthesize graphene oxide which then the obtained graphene oxide will be used as a filler of polymer graphene nanocomposite [7,10,11]. Modified Hummer's method is a versatile method that produce high level of oxidation without use complicated technique but this method takes longer time upon completion. Nevertheless, modified Hummer's method does not produce toxic as compared with other method such as Brodie and Staudenmaier method [3].

\section{Experimental}

\subsection{Materials and reagents}

Waste carbon tyre was supplied by Polis Di Raja Malaysia (PDRM) Cheras, Malaysia and was sieved to a particle size 200 $\mu \mathrm{m}$. The concentration of sulphuric acid $\left(\mathrm{H}_{2} \mathrm{SO}_{4}\right)$ and hydrogen peroxide $\left(\mathrm{H}_{2} \mathrm{O}_{2}\right)$ is $95-97 \%$ and $30 \%$. Potassium permanganate $(\mathrm{KMnO} 4)$ and sodium nitrate $\left(\mathrm{NaNO}_{3}\right)$ were slowly added to keep the temperature under $20^{\circ} \mathrm{C}$.

\subsection{Preparation of graphene oxide by modified Hum- mer's method}

$23 \mathrm{ml}$ of sulphuric acid $\left(\mathrm{H}_{2} \mathrm{SO}_{4}\right)$ was mixed with $1.0 \mathrm{~g}$ of sodium nitrate $\left(\mathrm{NaNo}_{3}\right)$ and stirred for several minutes. $1.0 \%$ by weight of waste carbon tyre powder was added into the solution under stirring condition. Then the mixture was transferred in the ice bath and stirred for 2 hours. $3.0 \mathrm{~g}$ of potassium permanganate $\left(\mathrm{KMnO}_{4}\right)$ was slowly added into the mixture and stirred for another 1 hour. The ice bath was removed and the mixture was leaved for 16 hours. The mixture was heated up to $35^{\circ} \mathrm{C}$ and stirred for another 1 hour. $46 \mathrm{ml}$ of distilled water was added into the mixture and stirred another 2 hours while heated up the mixture up to $95^{\circ} \mathrm{C}$. After 2 hours, the heater was switched off. $10 \mathrm{ml}$ of hydrogen peroxide $\left(\mathrm{H}_{2} \mathrm{O}_{2}\right)$ was added into the mixture and stirred for 1 hour to eliminate excess $\mathrm{KMnO}_{4}$ then let it cool to room temperature. $10 \mathrm{ml}$ of hydrochloric acid $(\mathrm{HCl})$ and $30 \mathrm{ml}$ of deionized water (DIW) was added into the solution and was centrifuged for 10 minutes at $8500 \mathrm{rpm}$ using Biofuge Stratos centrifuge. The supernatant was decanted away and rewashed again the residuals with $\mathrm{HCl}$ and DIW for three times. The washed GO solution was dried using oven at $70{ }^{\circ} \mathrm{C}$ for 24 hours.

\subsection{Preparation of graphene from graphene oxide by thermal exfoliation method}

Graphene synthesized from graphene oxide by thermal exfoliation method by heat up the dried graphene oxide (GO) rapidly. $2.0 \mathrm{~g}$ of GO was dried and charged into a quartz tube. Then, the graphene oxide as flushed with nitrogen gas for five minute. Quickly, the quartz tube was inserted into preheated furnace at $1100{ }^{\circ} \mathrm{C}$ and keep for several minutes to obtain graphene powder $[12,13]$.

\subsection{Materials characterization}

The morphology of the graphene oxide was analysed using a scanning electron microscope (SEM). It also used to determine morphological characterization. This microscopy also has been used for some observation such as topographical observation (secondary electron image), compositional observation (backscattered electron image), elemental composition observation using EnergyDispersive X-ray (EDX), and scanning electron microscope (SEM) analysis. SEM was used to provide morphological and elemental information with cover of magnification from low to higher magnification. For this sample, graphene oxide does not need any coatings for sample preparation. The sample was sprinkled on the carbon tape at the holder. The holder was placed into the SEM. The magnification of SEM that was used to observe the micro- graph of graphene oxide is 500x (low magnification) and 1000x (high magnification).

Raman spectroscopy was used to confirm the presence of graphene or otherwise. Single or few layer graphene will create at the range of $800 \mathrm{~cm}^{-1}$ to $2000 \mathrm{~cm}^{-1}$ in Raman shift. G-band and Dband can be determined, which is the shift in the G-band, indicates the oxidation of sample. Raman spectra extracted of the sample is relatively to the graphene oxide [14]. Raman spectroscopy is the most direct and non-destructive technique to describe the structure including the defects, the ordered and disordered structures of carbon material and was be perform to analyse the carbon structure. From the previous research, Raman spectra of graphene and graphene oxide presented similar basal structure profiles with characteristic D band at around $1334 \mathrm{~cm}^{-1}$ and $\mathrm{G}$ band at around $1590 \mathrm{~cm}^{-1}$. The $2 \mathrm{D}$ band in the graphene oxide indicated that all graphitic layer been oxidized [15]. The sample was placed on the glass slide and put under the Raman spectroscopy for characterization.

\section{Results and Discussions}

\subsection{Scanning Electron Microscopy (SEM)}

Scanning Electron Microscopy was provided the morphology and structural properties of graphene oxide. The electron beam interacts with the sample producing various signals that can be used to obtain information about surface morphology and composition. Fig. 1 shows the SEM micrograph of graphene oxide that obtained from waste carbon tyre via Hummer's method. Based on Fig. 1, the SEM micrograph of graphene oxide shows the sheet or flakes structure are stalked together on the surface. It also shows bumping pieces with coarse surface. Flakes provided can be clearly seen randomly stalked and uniformly.
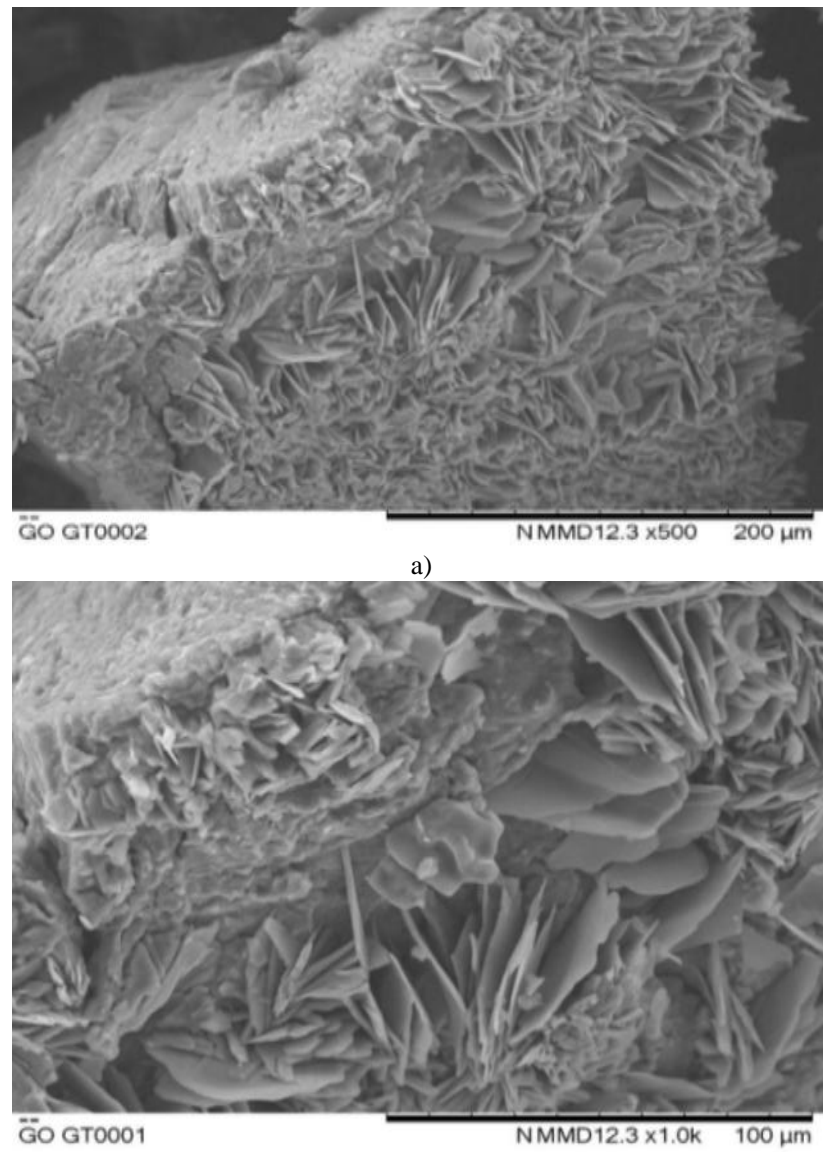

b)

Fig. 1: The SEM micrograph of graphene oxide obtained a) 500x magnification b) 1000x magnification 
Energy Dispersive X-Ray Spectroscopy (EDX) was used to measure the elemental analysis of graphene oxide. The Fig.2 shows the EDX result of graphene oxide. The oxygen and carbon that contain in graphene oxide is $49.10 \%$ and $50.90 \%$ respectively.

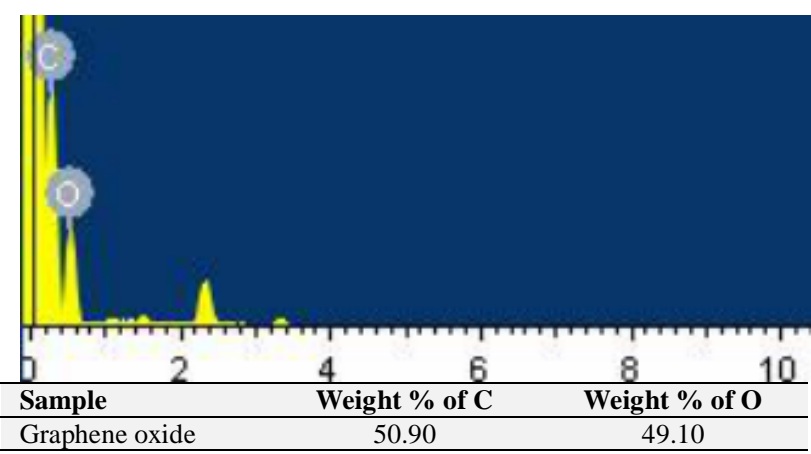

Fig. 2: The EDX image and composition table of graphene oxide

\subsection{Raman Spectroscopy}

Raman spectroscopy is non-destructive technique to characterize graphite material in particular to determine the defect, ordered, and disordered structure of graphene. This essentially identical to the characteristic peak of graphene oxide can be essentially identical by two peaks around $1500 \mathrm{~cm}^{-1}$ (D band and $\mathrm{G}$ band) [16]. Fig. 3 shows the Raman spectra of graphene oxide obtained.

Based on Fig. 3, the Raman spectra obtained display two peaks which at $1361 \mathrm{~cm}^{-1}$ and $1596 \mathrm{~cm}^{-1}$ corresponding to the well documented D-band and G-band. The band at $2434 \mathrm{~cm}^{-1}$ is known as the 2D band which is indicated that all graphite layers have been oxidized [15]. Compared to the Raman spectra from raw carbon tyre, the peak for D-band and G-band is $1339 \mathrm{~cm}^{-1}$ and $1581 \mathrm{~cm}^{-1}$. There is no peak for 2D band appears for raw carbon tyre. The intensity ratio of the two bands indicates the quality of product. The graphinization degree of carbon solid can be proved by the intensity ratio of two bands $\left(\mathrm{I}_{\mathrm{D}} / \mathrm{I}_{\mathrm{G}}\right)$ and lower value of ratio is present a high degree of graphinization [17]. In this research, the value of $I_{D}$ is 407 and the value of $I_{G}$ is 465 while the intensity ratio of the $\mathrm{D}$ band relative to the $\mathrm{G}$ band $\left(\mathrm{I}_{\mathrm{D}} / \mathrm{I}_{\mathrm{G}}\right)$ is 0.88 . The lower value of $\left(\mathrm{I}_{\mathrm{D}} / \mathrm{I}_{\mathrm{G}}\right)$ is indicated that it has low value of defect. Based on the result, it shows that grapheme oxide has been successfully produced [16].

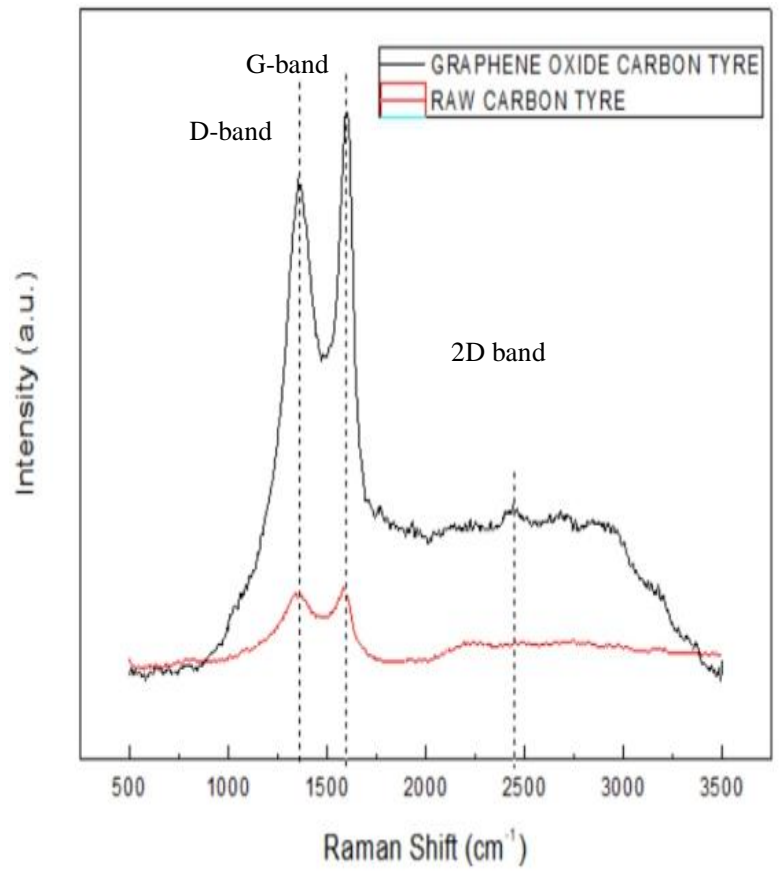

Fig. 3: The Raman spectra of graphene oxide obtained.

\section{Conclusions}

Graphene oxide successfully synthesized from waste tyre using modified Hummer's method. The structural properties of the graphene oxide obtained from modified Hummer's method successfully determined. Based on the scanning electron microscopy (SEM) and EDX characterization, the structure of graphene oxides are stalked together, bumping pieces with coarse surfaces and uniformly flake. The elemental composition of sample of $\mathrm{C}$ element is higher than $\mathrm{O}$ element which is it give a good composition of graphene oxide. On point of the Raman spectrum analysis, the Raman spectra that obtained from graphene oxide display two peaks, which is D-band, and G-band which at $1359.68 \mathrm{~cm}^{-1}$ and $1602.89 \mathrm{~cm}^{-1}$ respectively. There are 2D band appears in the spectrum at $2434 \mathrm{~cm}^{-1}$ which shows that graphite was oxidize. The value $\left(\mathrm{I}_{\mathrm{D}} / \mathrm{I}_{\mathrm{G}}\right)$ is 0.88 which indicate the successful of graphene oxide produced with low value of defect.

\section{Acknowledgement}

The authors would like to acknowledge Polis Di Raja Malaysia Cheras, Malaysia for providing waste carbon tyre for this research. We also would like acknowledge Faculty of Applied Sciences, Universiti Teknologi MARA (UiTM) Shah Alam for providing the financial support under project, 100-IRMI/INT16/6/2 (004/2018) and access to their research facilities and instruments. This research did not receive any specific grant from funding agencies in the public, commercial or not-profit-for sectors.

\section{References}

[1] Liu Y, Fan W, Xu Z, Peng W \& Luo S (2017), Comparative effects of graphene and graphene oxide on copper toxicity to Daphina magna : Role of surface oxygenic functional groups. Environmental Pollution NS, 1-9.

[2] Bhuyan MSA, Uddin MN, Islam MM, Bipasha FA \& Hossain SS (2016), Synthesis of graphene. International Nano Letter 6, 65-83.

[3] Lim JY, Mubarak NM, Abdullah EC, Nizamuddin S, Khalid M \& Inamuddin (2018), Recent trends in the synthesis of graphene and graphene oxide based nanomaterials for removal of heavy metals A review. Journal of Industrial and Engineering Chemistry NS $\mathrm{XX}-\mathrm{YY}$

[4] Zaaba NI, Foo KL, Hashim U, Tan SJ, Liu WW, \& Voon CH (2017), Synthesis of graphene oxide using modified Hummer's method : Solvent influence. Procedia Engineering 184, 469-477.

[5] Zhao JG, Xing BY, Yang H, Pan QL, Li ZP \& Liu ZJ (2016), Growth of carbon nanotubes on graphene by chemical vapor deposition. New Carbon Materials 31, 31-36.

[6] Foo ME, \& Gopinath SCB (2017), Feasibility of graphene in biomedical applications. Biomedicine \& Pharmacotherapy 94, 354361 .

[7] Park S, He S, Wang J, Stein A \& Macosko CW (2016), Graphenepolyethylene nanocomposites : Effect of grapheme functinalization. Polymer 104, 1-9.

[8] Muzyka R, Kwoka M, Smedowski L, Diez N \& Gryglewicz G (2017), Oxidation of graphite by different modified Hummers methods. New Carbon Materials 32(1), 15-20.

[9] Yuan R, Yuan J, Wu Y, Ju P, Ji L, Li H, Chen L, Zhou H \& Chen J (2018), Graphene oxide - monohydrated manganese phosphate composites : Preparation via modified Hummers method. Colloids and Surfaces A: Physiochemical and Engineering Aspects 574, 5663.

[10] Qiu Y, Wang J, Wang Z, Zhang M, Yao, Y \& Wei N (2016), Thermoplastic polyester elastomer nanocomposites filled with graphene : Mechanical and viscoelastic properties. Composite Science Technology 132, 108-115.

[11] Li H \& Xie XM, (2018), Polyolefin-functionalized graphene oxide and its GO/HDPE nanocomposite with excellent mechanical properties. Chinese Chemical Letters 29, 161-165.

[12] Singh MK, Bhatnagar A, Pandey SK \& Mishra PC (2017), Experimental and first principle studies on hydrogen desorption behavior of graphene nanofibre catalyzed $\mathrm{MgH}_{2}$. Hydrogen Energy 42, 960968. 
[13] Dao TD \& Jeong HM (2015), Graphene prepared by thermal reduction- exfoliation of graphite oxide : Effect of raw graphite particle size on the properties of graphite oxide and graphene. Material Research Bulletin 70, 651-657.

[14] Hosseinpour A, Abdizadeh H, \& Golobostanfard MR (2017), Comparing the electrophoretic deposition process of graphene oxides synthesized through different methods. Thin Solid Films 631, 118123.

[15] Rajaura RS, Srivastava S, Sharma V, Sharma P, Lal C, Singh M \& Vijay Y (2016), Role of interlayer spacing and functional group on the hydrogen storage properties of graphene oxide and reduced graphene oxide. International Journal of Hydrogen Energy 41, 9454-9461.

[16] Thema FT, Moloto MJ, Dikio ED, Nyangiwe NN, Kotsedi L, Maaza M \& Khenfouch M (2013), Synthesis and characterization of graphene thin films by chemical reduction of exfoliated and intercalated graphite oxide. Journal of Chemistry 2013, 1-6.

[17] Kim SG, Park OK, Lee JH, \& Ku BC (2013), Layer-by-layer assembled graphene oxide films and barrier properties of thermally reduced graphene oxide membranes. Carbon Letters 14, 247-250. 